Journal of Applied Fluid Mechanics, Vol. 2, No. 1, pp. 29-38, 2009.

Available online at www.jafmonline.net, ISSN 1735-3572, EISSN 1735-3645.

DOI: $10.36884 / \mathrm{jafm} .2 .01 .11853$

\title{
Experimental Study of a Thermal Plume Evolving in a Confined Environment: Application to Fires Problems
}

\author{
T. Naffouti ${ }^{1}$, M. Hammami ${ }^{1}$, M. Rebay ${ }^{2}$, and R. B. Maad ${ }^{1 *}$ \\ ${ }^{1}$ Laboratoire d'Energétique et des Transferts Thermique et Massique, Faculté des Sciences de Tunis, \\ Département de Physique, El Manar2, 2092, Tunis - Tunisia \\ ${ }^{2}$ UTAP - Laboratoire de Thermomécanique (EA 3082) Faculté des Sciences, 51687 Reims, France \\ Email:Rejeb.BenMaad@fst.rnu.tn
}

(Received January 16, 2008; accepted May 11, 2008)

\begin{abstract}
We propose to study the natural convection flow resulting from the interaction of a fire with walls that surround it. Indeed, when a fire occurs in a tunnel or in a tower block, it creates a thermal plume causing a heating of the neighbouring walls. This heating by thermal radiation of the walls creates a phenomenon of thermosiphon which interacts with the plume. To study this flow we simulated the problem at the laboratory where we placed a rectangular source heated by Joule effect at the entrance of an open-ended vertical canal. The flow visualization by laser plan and the exploration of the thermal and dynamic fields inside the canal enabled us to describe the flow structure. In order to better characterize this flow, we carried out a fine analysis by studying the spectra of temperature fluctuations. This spectral analysis allowed us to clarify the energetic evolution of the vortexes during their ascension and verify some known spectral laws.
\end{abstract}

Keywords: Turbulent natural convection, thermal plume, thermosiphon, spectral analysis, vortex, spectral laws.

\section{NOMENCLATURE}

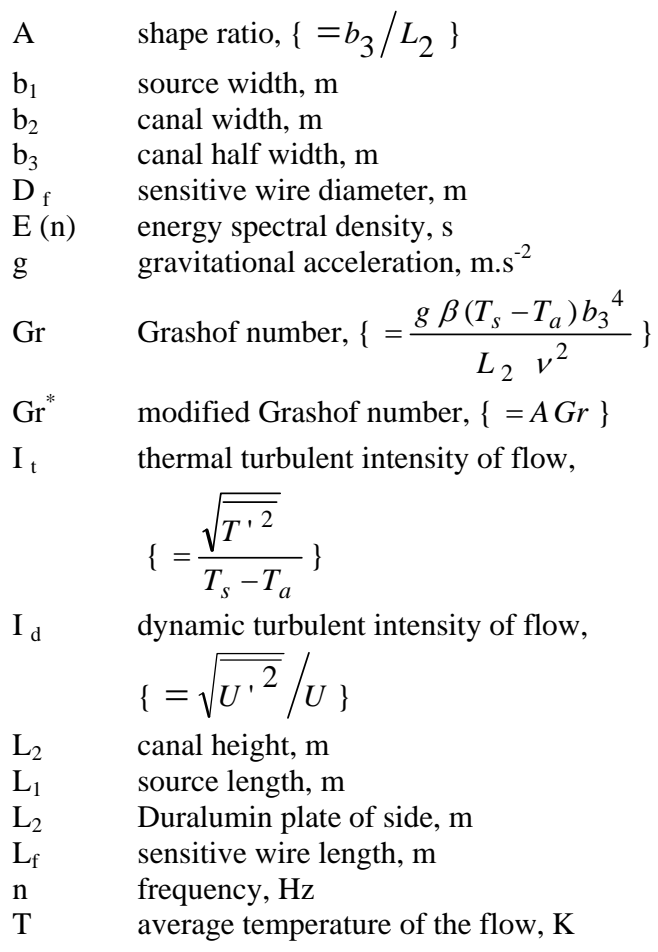

T’ temperature fluctuating, $\mathrm{K}$

$\mathrm{T}_{\mathrm{a}} \quad$ temperature of ambient air, $\mathrm{K}$

$\mathrm{T}_{\mathrm{s}} \quad$ temperature of the hot source, $\mathrm{K}$

$\mathrm{T}^{*}$ dimensionless average temperature of the flow, $\left\{=\frac{T-T_{a}}{T_{s}-T_{a}}\right\}$

$\mathrm{U} \quad$ average velocity of the flow, $\mathrm{ms}^{-1}$

U' velocity fluctuating, $\mathrm{ms}^{-1}$

$\mathrm{U}^{*}$ dimensionless average velocity of the flow, $\left\{=U / U_{\text {ref }}\right\}$

$\mathrm{U}_{\text {ref }} \quad$ reference velocity, $\left\{=L_{2} v\left(G r^{*}\right)^{\frac{1}{2}} / b_{3}^{2}\right\}$

$(\mathrm{x}, \mathrm{y}, \mathrm{z}) \quad$ Cartesian coordinates

$X^{*} \quad$ dimensionless coordinate, $\left\{=2 x / b_{2}\right\}$

Z* dimensionless height, $\left\{=z / L_{2}\right\}$

\section{Greek Letters}

$\begin{array}{ll}\beta & \text { thermal expansion coefficient, } \mathrm{K}^{-1} \\ v & \text { cinematic fluid viscosity, } \mathrm{m}^{2} \cdot \mathrm{s}^{-1}\end{array}$ 


\section{INTRODUCTION}

The study of a thermal plume is of crucial importance to understand the development of fire in unlimited environment. This kind of the flow encountered in fires of buildings or forests has been the object of several studies. J. Agator (1983) and Guillou (1983) studied the thermal plume induced by a spherical calotte heated at a uniform temperature of $500{ }^{\circ} \mathrm{C}$. They noted that the flow is divided in two different zones. In the first zone of a preestablishment of turbulence the plume continues to supply fresh air regularly during its ascent. This zone is characterized by high values of velocity and an important gradient of temperature. In the second zone of established turbulence, the plume extends and exported to the outside. The average and fluctuating thermal and dynamical profiles are flattened and self-similar. In addition, Brahimi (1987) studied experimentally the structure of a thermal plume generated by a disk heated electrically at $500{ }^{\circ} \mathrm{C}$. He noticed the appearance of the two zones described previously.

However, thermal plume generated by such fire interacts narrowly with the neighbouring walls. Indeed, thermal radiation emitted by the plume heats the walls which create a thermosiphon flow that is added to the thermal plume. The interaction of a plume with its material environment was the subject of several investigations. For example, Agator (1983) who studied a thermal plume in the presence of a second identical one or of a vertical plane wall placed in the vicinity, noted a strong interaction between the plume and the wall and the plumes evolving one near the other. By studying the interaction of two turbulent plumes of the same power, Brahimi (1987) showed the subdivision of the resulting flow in two distinct zones. He also noted that the evolution of the thermal and dynamic fields of high altitudes is similar to that observed in the established zone of the free plume.

Mahmoud et al. (1998), studied the interaction of a thermal plume with a thermosiphon which surrounds it. The thermal plume is produced by a flat disk heated by Joule effect at a temperature of $300^{\circ} \mathrm{C}$. This disk is placed at the entrance of an open-ended vertical cylinder of adiabatic wall. They noted the appearance of a supplementary zone near the hot source in addition to the two classic zones mentioned in the previous works concerning the free plume. Thereafter, Zinoubi et al (2004) continued this work by studying the influence of source-cylinder spacing and the cylinder height on the resulting flow. They noted a blocking of the ascending flow in the upper part of the cylinder due to the side expansion of the plume. Also, they noted an optimum position where the flow rate is increased and the flow temperature is homogenised at the system exit.

In order to better understand the evolution of this type of flow, several authors are interested by the spectral analysis of the themal and dynamic fluctuations. Doan-Kim-Son (1981, 1977) and Agator (1983) studied the temperature of spectra a natural convection flow produced by a hot source. In the first and the second zone of the flow, they noticed that the thermal spectra are centred respectively around 3.5 and 6.5
Hz. Also, they noted that the proportion of the big sizes vortexes decrease and those of the small sizes increase as we move away from the hot source. Moreover, they showed that the law $n^{-3}$ defined by Lumley (1964) is checked in the transition zone towards turbulence where the vortexes sizes are average. Thereafter, Brahimi (1987) analyzed the temperature spectra of the thermal plume. In the second zone of turbulence, he noticed that the maximum frequency of the flow is $5 \mathrm{~Hz}$ and the spectral energy is concentrated around $3 \mathrm{~Hz}$.

In 1995, Maad (1995) analyzed the spectra of temperature fluctuations of the natural convection flow in vertical heated canal. He noticed that the law $n^{-1}$ of Tchen (1953) is only observed at the entry of the canal where the production of energy is strong. In addition, he checked the existence of the law $\mathrm{n}^{-7}$ of Heisenberg at the exit of the canal where the viscosity forces are dominant (Doan-Kim-Son 1977).

By studying the interaction of a thermal plume with the thermosiphon flow which surrounds it, Zinoubi (2006, 2007) examined the spectra of the temperature fluctuations obtained by the fast Fourier transform inside a vertical cylinder. He noted the existence of the spectral laws introduced by Tchen (1953), Heisenberg (1977) and Lumley (1964). In addition, the universal law of Kolmogorov in $n^{-5 / 3}$ known in forced convection is not observed in any spectra of energy obtained in natural convection (Doan-Kim-Son 1981; Agator 1983; Zinoubi 2006).

In this work, to better understand the development of a fire in confined environment such as in tower blocks or in tunnels, we simulate the problem in the laboratory by studying the evolution of a thermal plume generated by a rectangular source placed at the entry of a parallelepipedic vertical canal. The study of this flow helps us to better control these fires.

\section{EXPERIMENTAL APPARATUS AND TECHNIQUES OF MEASUREMENTS AND TREATMENTS}

\subsection{Experimental Apparatus}

The experimental apparatus is represented in Fig.s 1a and 1b. The numbers in the description presented below refer to the part numbers of Fig.1. The thermal plume created by a rectangular flat source (1) is electrically heated by the Joule effect to a surface temperature of $300^{\circ} \mathrm{C}$. This source, which has a length of $L_{1}=0.42 \mathrm{~m}$ and a width of $b_{1}=0.06 \mathrm{~m}$, is placed horizontally at the entrance of the open ended vertical parallelepipedic canal (2). The uniformity of the source surface temperature is obtained by the use of wire resistors mounted behind the surface. A thermal regulation apparatus keeps the surface temperature as uniform as possible within a good approximation (the temperature difference between ends and in the middle is less than 1\%). In order to reduce the heat losses from below and minimise the temperature variation of the ambient air, this source is insulated at this side. The canal is constituted by two Duralumin (AU4G) square parallel flat plates of side $\mathrm{L}_{2}=$ 
$0.40 \mathrm{~m}$ and two rectangular Plexiglas plates $(0.40 \times 0.15$ $\mathrm{m}^{2}$ ). The system is placed on a frame at $0.80 \mathrm{~m}$ above the ground to allow air supply from below (3). The temperature of canal internal walls heated by thermal radiance of the hot source is controlled by thermocouples type- $\mathrm{K}$ introduced in the back of each plate. In a similar way, the canal walls are thermally insulated at the back to minimise heat losses. The strong dependence of the flow on the surrounding conditions requires conducting the experiment in a quiet atmosphere. To this effect, the experimental apparatus is placed in an independent closed room. In order to check the thermal stability of ambient air several thermocouples are fixed at different heights in the room. To explore thermal and dynamic average and fluctuating fields inside the canal, a resistant wire anemometer at constant current is used (4). Doan Kim-Son et al. (1981) adopted this technique for a long time in natural convection measurements. It is based on the principle of the resistance variation of a platinum wire. The sensitive platinum wire of the probe has a diameter of $\mathrm{D}_{\mathrm{f}}=7.5 \mu \mathrm{m}$ and a length of $\mathrm{L}_{\mathrm{f}}=0.003 \mathrm{~m}$.

In order to avoid the disturbance of the flow, the probe is introduced vertically through the system exit, so that its sensitive wire is perpendicular to the ascending average flow. A probe displacement system (6) in the $\mathrm{x}$ and $\mathrm{z}$ directions, is used to explore the thermal field inside the canal. The minimal displacement in the vertical direction is $1 \mathrm{~mm}$, whereas in the horizontal direction it is $0.20 \mathrm{~mm}$.

On the other hand, a computer provided with a data acquisition card permits to take the instantaneous measures of temperature and velocity and to record signals for further statistical processing $(5,7,8)$. In application of the Shannon theorem, the period of sampling of $15 \mathrm{~ms}$ was chosen.The visualisation system used during this study is represented in Fig.2. It is constituted of a He-Ne Laser with a power of 35 $\mathrm{mW}$ (1), an electric vibrant plate (2), a digital camera (5) and a smoke distributor (4). On the vibrant plate is glued a plane mirror of good quality, that receives a horizontal laser beam and it reflects according to the canal vertical axis. The displacement of the plate makes revolve the mirror around a horizontal axis creating a plane laser sheet (3) on the entire transverse section of the canal. By natural aspiration, the distributor allows smoke to impregnate all the flow without any disruption. Preliminary studies of visualization and measurements by the hot wire anemometry made it possible to notice that the smoke introduced at the entry of the system does not disturb the flow. To record video sequences from visualization a numerical camera (5) is used.

\subsection{Techniques of Measurements and Data Treatments}

To explore the thermal and dynamic fields of the fluid inside the canal, we used the technique of hot wire anemometry at constant current (CCA). The principle of this method is based on the resistance variation of the sensitive wire according to the temperature and velocity when it is supplied by a constant current (Doan-Kim-Son 1975; Morcos 1991).

The measurements of the thermal field are carried out by cold wire anemometer supplied by a current of $1.2 \mathrm{~mA}$ instead of using thermocouples. Indeed, the time-constant of the sensitive wire is $1 \mathrm{~ms}$ and that of thermocouple of $25 \mu \mathrm{m}$ in diameter is $50 \mathrm{~ms}$.

The hot wire anemometer is used to measure the local velocity of the flow. In this case the probe is supplied by a current of $50 \mathrm{~mA}$ which makes it sensitive simultaneously to the temperature and the velocity (Doan-Kim-Son 1981; Paulon 1987). The thermal inertia of the probe does not introduce any measurement errors especially at the low frequencies found in thermal plume (Agator 1983; Doan-Kim-Son 1981). The other sources of errors coming from the probe calibration are lower than $1 \%$ (Mahmoud 1998; Maad 1995).

In order to better characterize the turbulent flow, we carried out an analysis of the thermal energy spectra. For that, we passed to frequency space by the fast Fourier transform (FFT) applied to the autocorrelation function of the temporal signal (Doan-Kim-Son 1981; Lochet 1982). The Fig. 3 shows the statistical processing of the signal sampled by the probe.

\section{FLOW VISUALIZATION AND EXPERIMENTAL RESULTS}

\subsection{Flow Visualisation}

For constraints of laser source power the visualization of the flow by a laser plan was sometimes limited to the half width of the vertical canal (Figs. 5 and 7).

The visualization performed by illuminating the flow by a laser plan sheet reveals the evolution of the flow from the canal entry to its exit. Some instantaneous photos and drawings from the video recording are presented in Figs. 4-7.

The Figs. 4 and 5 show that the system is supplied in fresh air only from below. The fresh air, aspired on both sides of the hot source, is divided in three fluid threads. The first thread and its symmetrical in relation to the longitudinal median plan, pursues its path directly towards the central part of the flow to shape an envelope above the hot source. This impenetrable envelope at the sides obliges the fresh air arriving from the low to get in by its top. The trapped fluid near the source heats up rapidly by the contact of the hot surface. After, it goes up again under the effect of buoyancy forces and forms a big vortex, which escapes vertically (Fig. 6). To replace this released fluid, this region is supplied again with fresh air that will undergo the same process. However, we note the existence of a certain alternation between the formation and the exhaust of these vortexes on both sides of the median plan (Fig. 6). Indeed, these two mechanisms do not occur simultaneously; as one of the vortexes escapes at the side, the other forms at the further side.

The second thread, relatively near to the canal wall, undergoes at the start a strong attraction by the plume before coming back to feed the flow along the canal walls. On the other hand, the Fig. 5 showed that this thread is entirely influenced by the formation-exhaust cycle of the vortexes. 
In fact, when the vortex exhausts, the thread is repelled toward the wall to continue its way along this one. Simultaneously, the symmetrical thread undergoes the important attractive effect of the plume during the formation of the vortexes.

The third thread constitutes a provision source in fresh air for the flow. By its intermediate position between the two threads described previously, this one undergoes an interaction with the plume and the canal wall (Figs. 6 and 7). In fact, this thread is also submitted, as previously, to the same mechanisms of formation and exhaust of the vortexes. After that, this one pursues its way directly toward the central part of the flow to from a vortex, which ends by escaping vertically (Figs. 6 and 7).

Elsewhere, we remarked that the flow ascending undergoes a transversal contraction to a height neighbouring $10 \mathrm{~cm}$ above the hot source (Fig. 7). Thereafter, it enlarges towards the walls after which vortexes of much smaller sizes occupy the entire upper section of the canal.

In the third zone, the visualisation shows the absence of the ascending flow blocking noticed by Zinoubi (2004) in the cylindrical geometry study.

This qualitative description enables us to suppose the existence of three zones which characterize the vertical evolution of the flow inside the canal (Fig. 7). In the first zone, we attend the development of a flow strongly influenced by the hot source. This behaviour changes completely in the second zone where the contraction takes place, whereas the flow becomes homogeneous in the upper part of the canal.

\subsection{Experimental Results}

The results which we present here are carried out with air. The Grashof number of the flow is $\mathrm{Gr}=0.3110^{7}$ and the shape ratio is $A=0.18$. The results are presented for two sections of study in each one of the three zones (Fig. 8).

\subsubsection{Evolution of the Average Temperature}

The Fig. 9 shows the distribution of the dimensionless average temperature of the flow for six transversal sections. Therefore, we can distinguish three different evolutions of the flow. Indeed, in the vicinity and above the hot source $\left(Z^{*}=0.05, Z^{*}=0.10\right)$, the fresh air warms up quickly and reaches important temperatures constituting a stage. On both sides of this stage, we note high thermal gradients translating a brutal transformation of the fresh air into hot plume. In intermediate space between the source and the vertical walls of the canal, the fresh air dominates the flow except that close to the walls where we record a light rise of temperature due to the heating of these last by thermal radiation of the hot source. The profiles relating to the intermediate zone $(\mathrm{Z} *=0.20, \mathrm{Z} *=0.32)$ show an attenuation of the temperature in the central region of the canal accompanied by a contracting of the stage mentioned previously. In the third zone $(\mathrm{Z} *=0.75, \mathrm{Z} *=0.92)$, the thermal profiles become self-similar and the temperature remains practically constant in all the section. This homogenisation of the temperature of the flow is due to the establishment of turbulence in the superior part of the canal.

\subsubsection{Evolution of the Average Velocity}

The transverse distribution of the dimensionless average velocity of the flow is represented in Fig. 10. The general pace of these profiles confirms the existence of the three zones announced previously. Indeed, in the first zone near to source $(\mathrm{Z} *=0.05, \mathrm{Z} *=0.10)$, the profiles show very strong velocity gradients on both sides of the hot source. This is due to the existence of the feeding threads wrapping the plume. The minimum of velocity observed in the vicinity of the median plan of the source translates the existence of a weak circulation region of the fluid.

In second zone $(\mathrm{Z} *=0.20, \mathrm{Z} *=0.32)$, the profiles show a strong velocity in the central part of the canal due to the flow contraction.

In the upper part of canal ( $\mathrm{Z} *=0.75, \mathrm{Z} *=0.92)$, the profiles of velocity become practically similar thus indicating the mode establishment in this zone.

\subsubsection{Thermal Turbulent Intensity}

The thermal intensity of turbulence represented in Fig. 11, for the various sections of study $\left(0.05 \leq Z^{*} \leq 0.92\right)$, confirms the existence of the three zones of the flow announced previously.

In the zone close to the hot source surface $\left(\mathrm{Z}^{*}=0.05, \mathrm{Z}^{*}=\right.$ 0.10 ), the profiles shows the existence of a minimum on the median plan of the canal and two maximum on both sides of this one. The minimum of the turbulent intensity is due to the predominance of the plume. The peaks of the rates of turbulence, corresponding to the region of the flow where the transverse heat gradients are maxima, are due to the strong interaction of the plume with the feeding fresh air. Near the walls of the canal, we note relatively low rates of turbulence.

In addition, in the second zone of the flow $(Z *=0.20$, $Z *=0.32$ ), we note that the turbulent rate increases in the central region above the source. The existence of the maximum of turbulent rate is related to the cycle formation and exhaust of the vortexes of the central envelope described by the interpretation of visualization.

In the third zone $(\mathrm{Z} *=0.75, \mathrm{Z} *=0.92)$, the profiles of thermal intensity of turbulence self-preserve thus indicating the establishment of turbulence in all the higher part of the canal.

\subsubsection{Dynamic Turbulent Intensity}

The profiles of the dynamic turbulent intensity are represented in Fig. 12. These profiles show also the evolution of the resulting flow in different zones. For the levels close to source $(\mathrm{Z} *=0.05, \mathrm{Z} *=0.10)$, these profiles show the existence of three extreme in the vicinity of the median plane. The maximum of turbulence of $40 \%$ corresponds to a strong interaction between the plume and 
the feeding threads coming from the ambient environment. The minimum of dynamic turbulence on the median plane is due to a weak circulation of the resulting flow inside the envelope described previously. In the intermediate zone $(Z$ $*=0.20, \mathrm{Z} *=0.32$ ), the profiles show a reduction of the turbulent intensity to $30 \%$ in the central part of the flow thus indicating the pre-establishment of turbulence. In addition, the dynamic turbulence rates increase to $40 \%$ near the walls of the canal. This increase of turbulence shows a strong interaction between the feeding threads that persist in this zone and the vortexes exhaust. At the higher levels $(\mathrm{Z}$ * $\left.=0.75, Z^{*}=0.92\right)$, the rates of dynamic turbulence are stabilized in the vicinity of the median plane source and the profiles are self-preserved thus indicating the establishment of the turbulent mode.

\subsubsection{Spectral Analysis of the Temperature Fluctuations}

To better define the fine structure of the resulting flow plume-thermosiphon, a deep study of the thermal energy spectra is necessary. Indeed, this will enable us to specify the energy contribution of the temperature fluctuations.

The spectra of thermal energy, obtained by using the fast Fourier transform (FFT), are standardized in the following form: $\int_{0}^{\infty} E(n) d n=1$ where $\mathrm{n}$ indicates the frequency.

According to the previous works, the hypothesis of equivalence space-time of Taylor is not checked in turbulent natural convection (Doan-Kim-Son 1981; Agator 1983). For that, in this study, the spectra of energy are presented according to the frequency instead of the number of wave as it is often the case in forced convection.

Because of the resemblance which we noticed between the spectra in each zone of the flow, the spectral analysis of temperature fluctuation was limited to the three sections of study $\left(Z^{*}=0.10, Z^{*}=0.20\right.$ and $\left.Z^{*}=0.75\right)$. The spectra of the temperature fluctuation are presented for three fixed points located on the median plan $\left(X^{*}=0\right)$, at the edge of the source $\left(X^{*}=0.4\right)$ and close to the vertical wall $\left(X^{*}=\right.$ $0.96)$, and a variable point corresponding to the maximum of the thermal turbulent intensity $\left(\mathrm{X}^{*}=\mathrm{X}_{\mathrm{I} \max }\right)$.

The Figs. 13.a, b and c which represent the evolution of spectres of the temperature fluctuations, show the existence of a peak more or less important at the frequency $0.10 \mathrm{~Hz}$. This peak, corresponding to a big vortex, characterizes feeding the threads described by the flow visualization (Maad 1995).

In the first zone $Z^{*}=0.10$, the Fig. 13.a show the existence of other secondary peaks of a weaker energy at frequencies lower than $3 \mathrm{~Hz}$. These peaks are the consequence of the interaction of vortexes generated by the plume and the fresh air, which descends the envelope. At the edge of the source, the spectre shows the appearance of peaks of energy at frequencies higher than that relating to the peak corresponding to the feeding threads. Elsewhere, close to the canal wall, we notice the displacement of the principal peak at the frequency $0.25 \mathrm{~Hz}$, due to the interaction undergone by the feeding thread from the plume and the canal wall mentioned previously in the flow visualization. In the intermediate zone $\left(Z^{*}=0.20\right)$, the Fig. 13.b shows the appearance of a principal peak at 1.66 $\mathrm{Hz}$ accompanied by other secondary peaks with a comparable energy. These peaks are due to the exhaust of vortexes from the envelope towards outside. Elsewhere, for $X^{*}=0.96$, the spectre shows the presence of the peak at $0.10 \mathrm{~Hz}$ which indicates the persistence of the feeding thread in higher levels. In the third zone of the flow $\left(\mathrm{Z}^{*}=\right.$ 0.75), Fig. 13.c shows, in addition to the peak of $0.10 \mathrm{~Hz}$, the existence of other secondary peaks at higher frequencies. These frequencies indicate the predominance of dissipative vortexes characteristic of a turbulent flow.

On the Figs. 14.a, b and c, the spectra of the temperature fluctuations are represented in a semi-logarithmic coordinate system for the same points of the study. This representation permits the setting in evidence of the spectral strips containing a considerable energy, while taking in consideration the delimited areas by the spectra (Fulachier 1972). The transversal evolution of these spectra shows that most of energy is concentrated in a rather broad frequency band ranging between 1 and $10 \mathrm{~Hz}$ (Fig. 14).

For the zone close to the source $\left(Z^{*}=0.10\right)$, the Fig 14.a shows an important peak which moves from $2.3 \mathrm{~Hz}$ to 4.6 $\mathrm{Hz}$ going from the median plan towards the hot wall of the canal. This indicates that the proportion of the big vortexes increases in front of that of the small structures as we approach the median plan. In the second zone of the flow $\left(Z^{*}=0.20\right)$, the Fig 14.b shows an energy strip around 5 $\mathrm{Hz}$, frequency which characterizes the transition towards turbulence (Doan-Kim-Son 1981; Agator 1983). On the other hand, when we move away from the hot source of the plume, we notice that the proportion of energy corresponding to the middle size vortexes increases in front of those of the great size which occur at the entry of the system (Doan-Kim-Son 1981; Elicer-Cortés 2003; Zinoubi 2006). Indeed, the vortexes of big sizes carriers of energy, which depend on the conditions of entry, undergo a stretching in the direction of the ascending flow and give rise to vortexes of intermediate structures. During their progression, the later produce vortexes of lower size. In the higher zone of the canal $\left(\mathrm{Z}^{*}=0.75\right)$, a general displacement of the spectra of energy towards the high frequencies is noted (Fig 14.c), thus indicating the predominance of the small dissipative vortexes where the flow reaches a fully turbulent mode.

The representation of these spectra on a logarithmic scale (Figs. 15.a, b and c), us made it possible to show the absence of the universal law $n^{-5 / 3}$ of Kolmogorov. This classical law, observed in forced convection, does not verified in any spectra of temperature because the Reynolds values of the flow is low (Doan-Kim-Son 1981; Agator 1983; Zinoubi 2006; Zinoubi 2007).

In addition these spectra of thermal energy enable us to find some laws in power known in natural convection such as: 
- The spectral law in $\mathrm{n}^{-1}$ of Tchen (1953), valid in the region where the turbulent production of energy dominates the flow, is observed only in a narrow strip of frequency. This law is observed by several researchers in natural convection (Maad 1995; Zinoubi 2006; Zinoubi 2007).

- The spectral law in $\mathrm{n}^{-3}$ introduced by Lumley (1964) is rather well checked especially in the second zone of the flow. In this zone of transition towards turbulence (Agator 1983; Doan-Kim-Son 1981; Zinoubi 2006; Zinoubi 2007), the size of the vortexes seems to be intermediate between that of the large structures carrying energy, which occur at the entry of the system, and that of the small dissipative vortexes, which develop downstream.

- The law in $\mathrm{n}^{-7}$ of Heisenberg (Doan-Kim-Son 1977) is checked at higher frequencies and particularly in the third zone where the viscous forces dominate the flow.

\section{ConCLUSIONS}

This experimental work is a contribution to the comprehension of a fire behaviour which evolves in confined environment. This confinement causes appreciable changes of the fire global structure.

Flow visualization by laser plan and study of thermal and dynamic fields shows the appearance of a supplementary zone that is added to the two classical ones encountered in a free thermal plume. This zone of instability located just above the generating source is characterized by the formation of an impenetrable envelope. In addition, the results of this study show that the thermosiphon intervenes to limit the side expansion of the plume, activates the ascending flow and quickly homogenizes the fluid at the exit of the system. The analysis of the temperature fluctuations spectra shows that the big structures vortexes carriers of energy evolve toward smaller dissipative structures as we move away from the source. Indeed, the vortexes of big sizes which depend on the conditions of entry undergo a stretching in the direction of the ascending flow and give rise to vortexes of intermediate structures. During their progressions, these ones produce vortexes of lower size. This evolution is accompanied by a transfer of energy towards increasingly small vortexes until the viscous forces dominate the flow. On the other hand, this study allowed the checking of some known spectral laws in natural convection: the $\mathrm{n}^{-1}$ law of Tchen in the first zone of the flow, the $\mathrm{n}^{-3}$ law of Lumley in the intermediate zone and the $\mathrm{n}^{-7}$ law of Heisenberg in the last zone. The information obtained within the framework of this study can enable us to understand the development mechanism of fires in a confined environment. This information will help us develop techniques of prevention and to better control these fires.

\section{REFERENCES}

Agator, J.M. and K.S. Doan (1982). Turbulent structure of axisymetric thermal plume. Mech. Rec. Commun., 159164.
Agator, J.M. (1983). Contribution à l'étude de la structure turbulente d'un panache thermique à symétrie axiale. Interaction du panache avec son environnement limité, Thèse. Université de Poitiers.

Agator, J.M. and D.K. Son (1983). Analyse spectrale du champ thermique d'un panache turbulent à symetrie axiale. C. R. Acad. Sc. Paris 296(II), 1119-1122.

Brahimi, M. (1987). Structure turbulente des panaches thermique-interaction. Thèse, Université de Poitiers.

Doan, K.S., M. Stage, and J. Coutanceau (1975). Transfert de chaleur entre un fil anémométrique court et un écoulement à faible vitesse. Revue Générale de Thermique, 168.

Doan, K.S. (1977). Contribution à l'étude de la zone de transition et de la zone de turbulence établie dans un écoulement de convection naturelle sur une plaque plane verticale isotherme. Thèse de Doctorat d'Etat, Université de Poitiers.

Doan, K.S. and J. Coutanceau (1981). Structure d'un écoulement de convection naturelle-transition et turbulence établie. Acta Astronautica 8, 123-160.

Elicer-Cortés, J.C., R. Contreras, D. Boyer, M. Pavageau and R.H. Hernandez (2003). Temperature spectra from a turbulent thermal plume by ultrasound scattering. Experimental thermal and fluid science 28 (8), 803813.

Fulachier, L. (1972). Contribution à l'étude des analogies des champs dynamique et thermique dans une couche limite turbulente Effet de l'aspiration. Thèse de Doctorat d'Etat, Université de Marseille.

Guillou, B. and K.S. Doan (1983). Etude théorique de développement d'un panache thermique à symétrie axiale Influence des propriétés thermophysique du fluide. Int. Comm. Heat Mass Transfer 10, 101-109.

Lochet, R. (1982). Contribution à l'étude des corrélations spatio-temporelles des fluctuations de vitesse et de température en convection naturelle turbulenteInfluence de la pression et de la nature du gaz. Thèse de Docteur Ingénieur, Université de Poitiers.

Lumley, J.L. (1964). The spectrum of nearly inertial turbulence in a stably stratified fluid. J. Atm. Sci. 21, 99-102.

Mahmoud, A.O.M., R.B. Maad, A. Belghith (1998). Interaction d'un écoulement de thermosiphon avec un panache thermique à symétrie axiale: étude expérimentale. Rev. Gén. Therm. 37, 385-396.

Morcos, N.G. (1991). Techniques modernes de mesure anémométrique. Thèse de Doctorat d'Etat, Faculté des sciences de Tunis. 
Paulon, J. (1987). Evolution récente de l'appareillage et techniques de mesure en aérodynamique et en hydrodynamique. La Houille Blanche 6.

Rejeb, B.M. (1995). Etude d'un ecoulement de convection naturelle dans un canal vertical chauffé. Thèse de Docteur D’Etat ES-Sciences Physiques, Université de Tunis El Manar.

Tchen, C.M. (1953). On the spectrum of energy in turbulent shear flow. J. of Res. of the Nat. Bureau of Standards 50 (1), 51-62.

Zinoubi, J., R.B. Maad, and A. Belghith (2004). Influence of the vertical source-cylinder spacing on the interaction of thermal plume with a thermosiphon flow: an

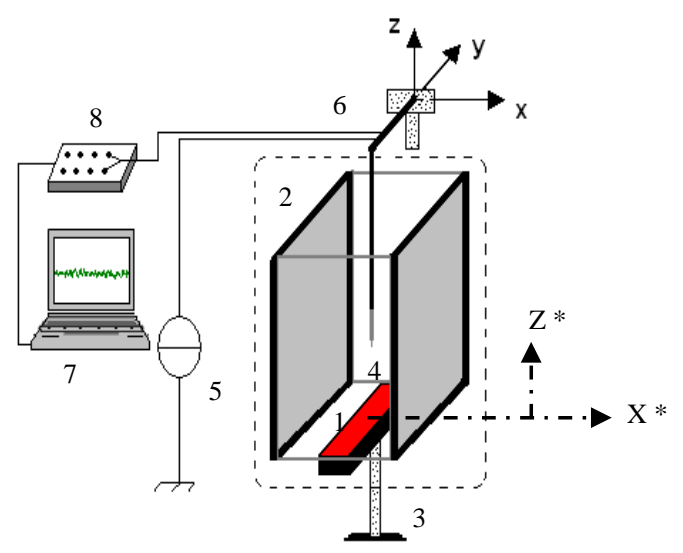

Fig. 1.a. Experimental apparatus 3D.

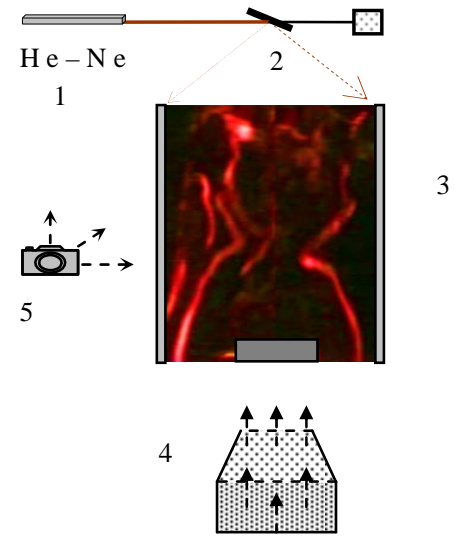

Fig. 2. Flow visualization system. experimental study. Experimental Thermal and Fluid Science 28, 329-336.

Zinoubi, J., A.O.M. Mahmoud, T. Naffouti, R.B. Maad, and A. Belghith (2006). Study of the Flow Structure of A Thermal Plume Evolving in an Unlimited and in A Semi-Enclosed Environment. American Journal of Applied Sciences 3 (1), 1690-1697.

Zinoubi, J., A. Gammoudi, T. Naffouti, R.B. Maad, and A. Belghith (2007). Development of an Axisymetric Thermal Plume between Vertical Plates. American Journal of Applied Sciences 4 (9), 697-685.

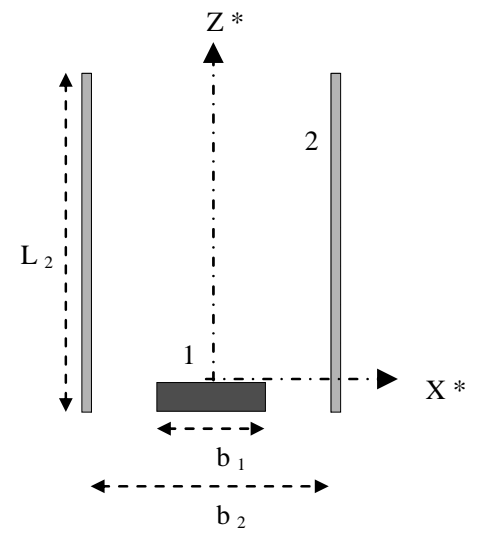

Fig. 1.b. Experimental apparatus 2D.

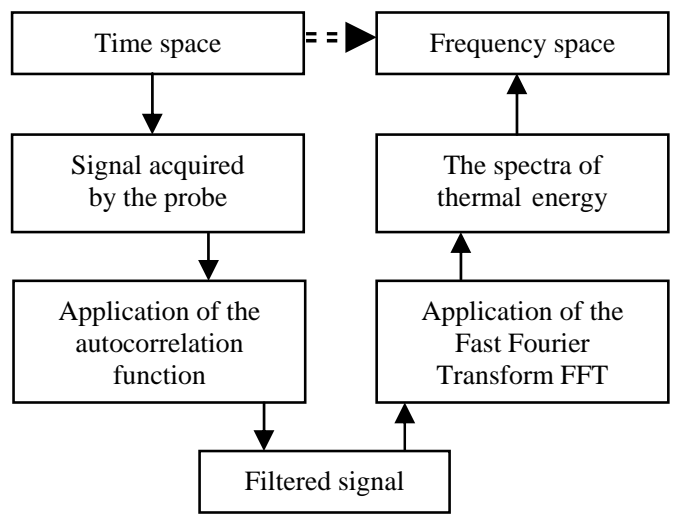

Fig. 3. Statistical processing. 
T. Naffouti et al. / JAFM, Vol. 2, No. 1, pp. 29-38, 2009.

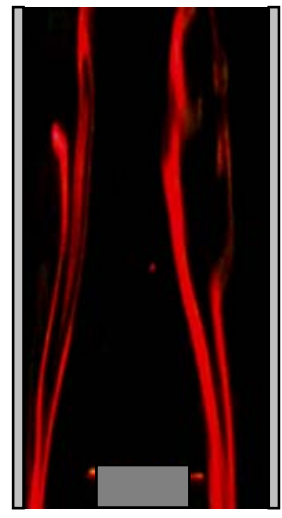

Fig. 4. The feeding threads.

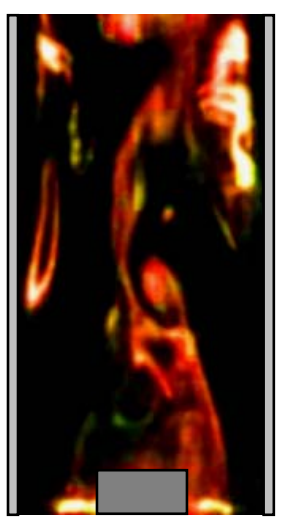

Fig. 6. Exhaust of the left vortex.

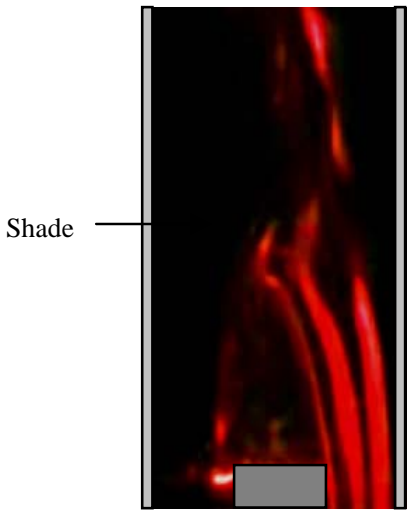

Fig. 5. The three fluid threads.

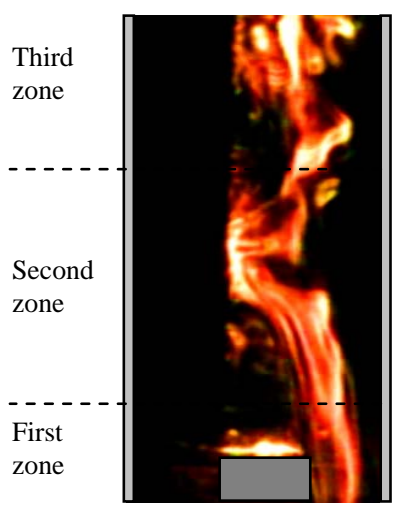

Fig. 7. Contraction and expansion of the plume.

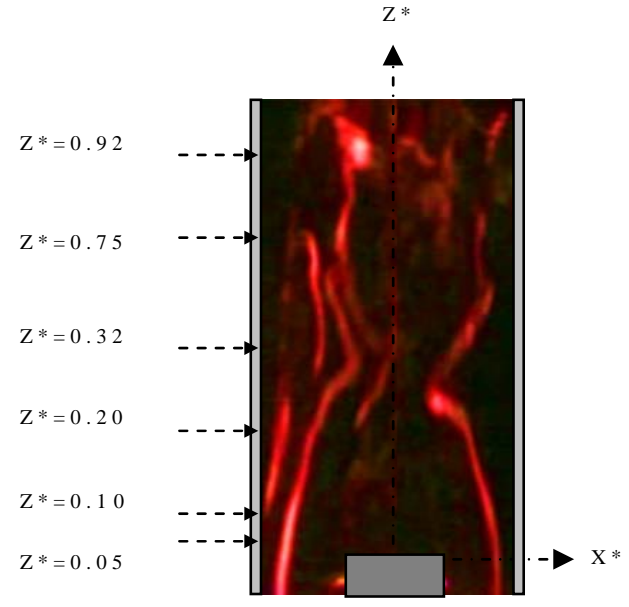

Fig. 8. Study sections 
T. Naffouti et al. / JAFM, Vol. 2, No. 1, pp. 29-38, 2009.

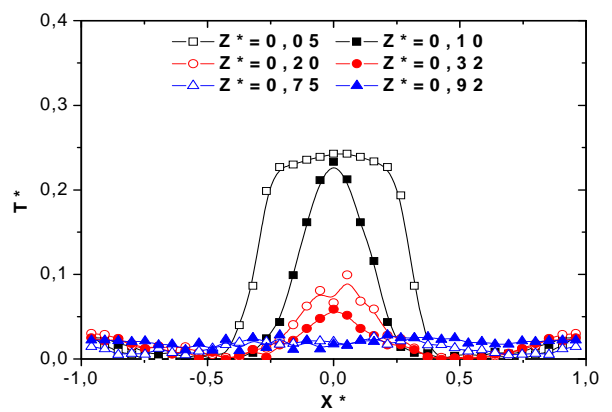

Fig. 9. Transversal evolution dimensionless average temperature.

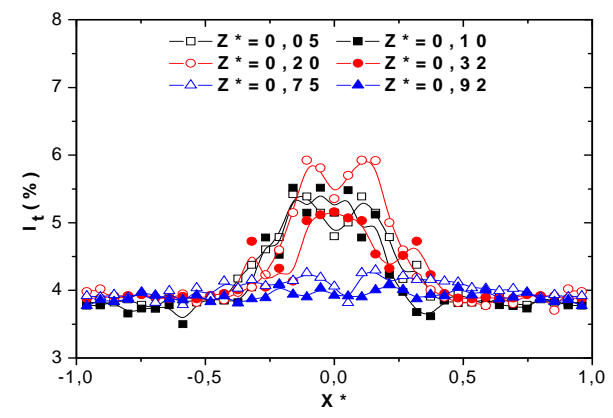

Fig. 11. Transversal evolution of thermal turbulent intensity.

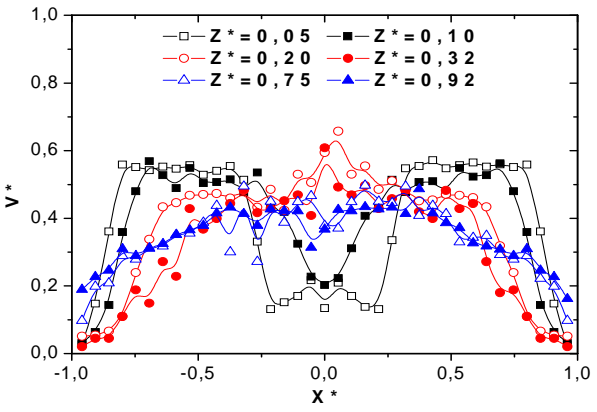

Fig. 10. Transversal evolution dimensionless average velocity.

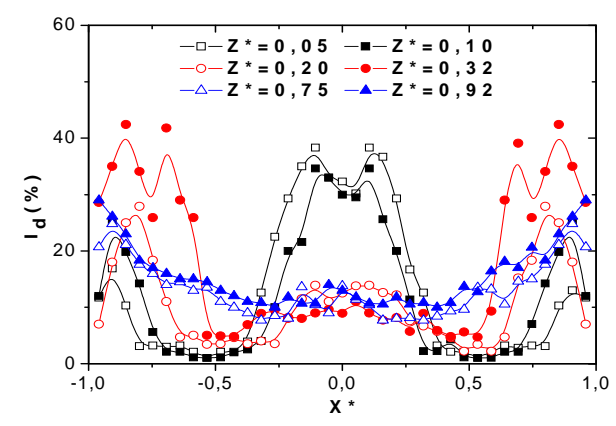

Fig. 12. Transversal evolution of dynamic turbulent intensity.
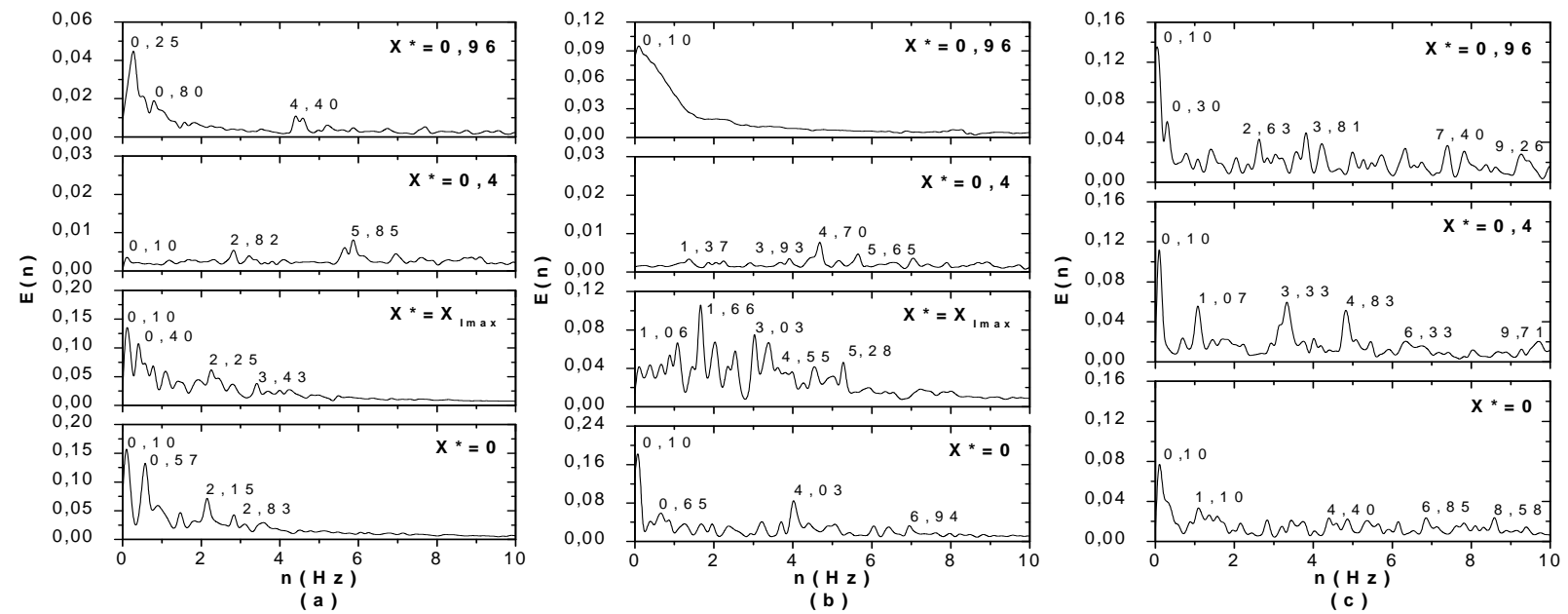

Fig. 13. Spectra of temperature fluctuations: a) $\mathrm{Z}^{*}=0.10$, b) $\mathrm{Z}^{*}=0.20$, c) $\mathrm{Z}^{*}=0.75$. 
T. Naffouti et al. / JAFM, Vol. 2, No. 1, pp. 29-38, 2009.

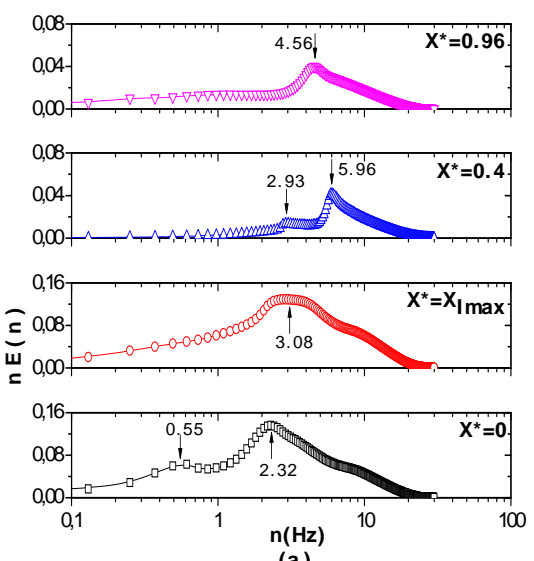

(a)
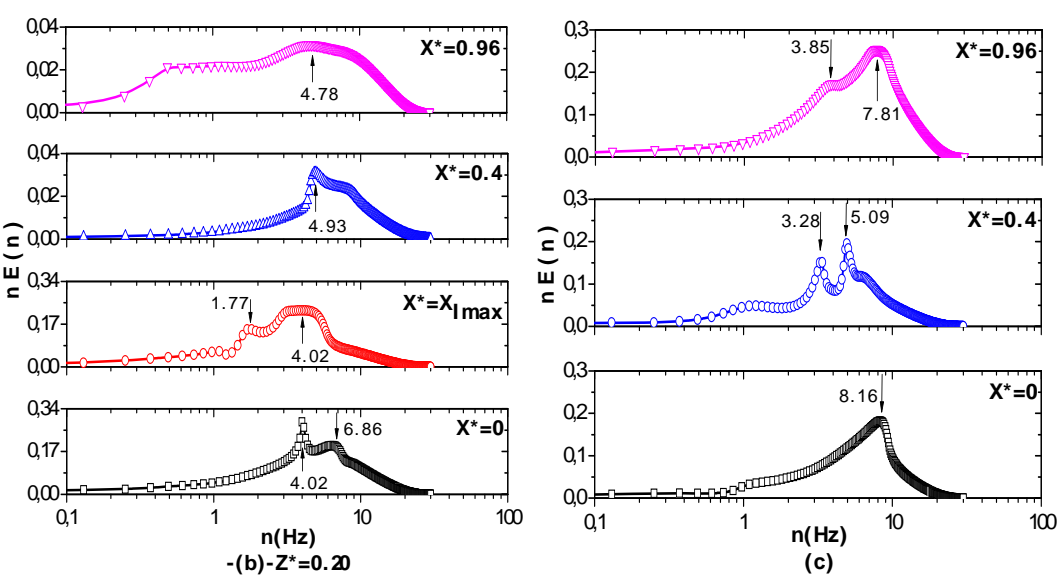

(c)

Fig. 14. Energy spectral density of the temperature fluctuations (semi-Log): a) $Z^{*}=0.10$, b) $Z^{*}=0.20$, c) $Z^{*}=0.75$.

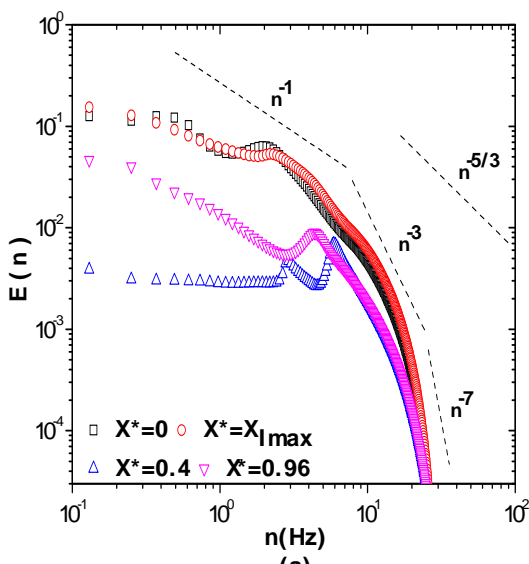

(a)

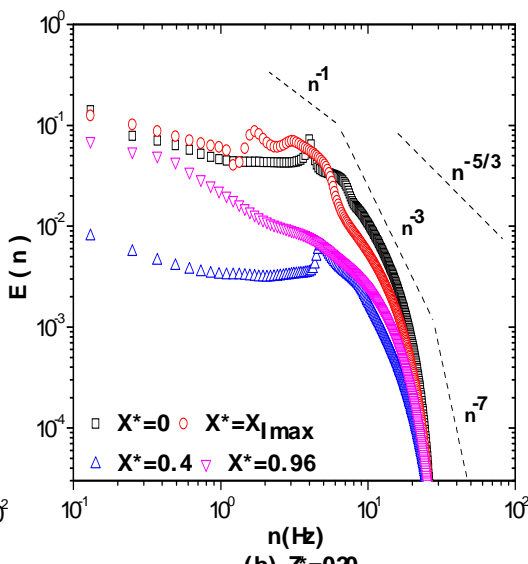

$-(b)-Z^{*}=0.20$

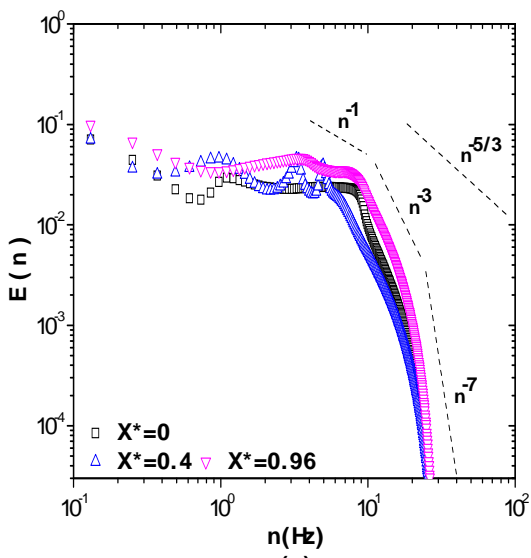

(c)

Fig. 15. Energy spectral density of the temperature fluctuations (Log-Log): a) $Z^{*}=0.10$, b) $Z^{*}=0.20$, c) $Z^{*}=0.75$. 